\title{
IMPACT OF LANGUAGE BARRIERS ON ACCESS TO HEALTHCARE SERVICES BY IMMIGRANT PATIENTS: A SYSTEMATIC REVIEW
}

Sasan Rasi

Griffith University, Queensland, Australia

Correspondence: sasanrasi@gmail.com

\begin{abstract}
BACKGROUND

Research has demonstrated lower access to healthcare services by immigrant patients in comparison to native people. Cultural and linguistic differences have been considered as main factors that impede this access and as barriers to creating an effective relationship between immigrant patients and health professionals.
\end{abstract}

\section{OBJECTIVE}

The aim of this study was to better understand and synthesize the available evidence regarding the impact of immigrant patients' language proficiency on access to health care.

\section{METHODS}

A systematic literature search was performed to identify studies published between January 2000 and January 2019 that examined the impact of language proficiency on access to and use of health services by immigrant patients. Only articles in English were included. Cross-referencing of the identified articles was also performed.

\section{RESULTS}

A total of 140 publications was identified through online databases. In all 24 studies were reviewed, and the results were reported using four interrelated themes identified from the articles. These reports consistently showed a clear association between inadequate language ability and underuse of health care services, ineffective communication, and increased use of emergency care by immigrant patients. Identifying factors that can influence access to care, applying immigrant-friendly solutions such as provision of professional interpreters, and encouraging culturally and linguistically sensitive education may improve the quality of care and increase access to care. One study recommended utilisation of communication technologies such as telemedicine to bridge the communication gap and increase accessibility of healthcare services by immigrant patients.

\section{CONCLUSIONS}

All included studies indicated that language barriers hindered access to healthcare services. The data resulting from this study can update policy and practical solutions for language barriers on access to care by immigrant patients and provide an agenda for further investigations.

\section{KEYWORDS}

language, healthcare services, access, barriers, immigrants, migrants

\section{INTRODUCTION}

The significant number of migrants around the world is a fact. [1] As the globalisation process increases, the migration rate of individuals from different cultures and countries is rising. Reasons such as more secure employment, better education, escape from war and disasters or reuniting with families can compel individuals to migrate. [2] Based on a United Nations report, the number of international immigrants reached 244 million in 2015. [3]

Legal immigrants in host countries have the same access to health care services as native-born. However, inequalities in access to health care services have been reported. [4] 
Access to healthcare services and facilitating this access is defined as helping people to command appropriate health care resources in order to preserve or improve their health. [5]

To gain access, patients need to be able to proficiently speak in the same language of the health professionals who provide the care. This proficiency is particularly vital in dealing with medical conditions, because clinical examinations depend on verbal communication between patients and medical staff. [6, 7] This miscommunication also increases the risks of delays in treatment, inadequate care, medical errors, and misdiagnosis received by these patients. Potential drug-related adverse effects and medical complications are also reported. [7-9]

Language barriers have been shown in various research studies as a significant impediment in establishing an effective doctor-patient relationship. [9-12] Barriers to communication between immigrant patients and health professionals can affect patients' access to available information or understanding of the information received. These barriers can also affect their decisions to accept and adhere to some types of treatment and medications. [1317]

Australia has a long history of immigration' more than many other countries and is well recognised as a multi-cultural nation. According to Commonwealth of Australia statistics, Australia is a popular destination for immigrants and hosts one of the largest immigrant populations in the world. The latest trend data in Australia, shows that approximately $29 \%$ (7.3 million) of the Australian population was born overseas as at June 2018. [18]

There has been an increase in cultural diversity in Australia with more than 200 cultural and linguistic groups. [19] In this diverse background, the Australian health care system is experiencing a growing difficulty in delivering healthcare in an equal access and culturally capable manner. [20]

This review study focuses on the challenge of providing quality care in the context of immigration to developed countries such as Australia and the impact of language barriers on access to health care by immigrant patients.
The objective of this review is to synthesize the current evidence and to gain better understanding of language barriers, which is currently placing immigrants and patients from different ethnicities at high risk of suffering poorer health services.

The review is also searching for new and advanced solutions that can minimise the risk and fill the gaps in access to quality health care services by immigrants and ethnic minority groups. These solutions can apply to the Australian healthcare system.

\section{METHODS}

This review is steered by the Arksey and O'Malley framework for performing scoping review. The framework for performing scoping review has five stages. The fivestage framework includes: 1) identify the research question (s), (2) identify relevant studies, (3) select the study, (4) chart the data, and (5) report the results. [21]

Scoping reviews use systematic methods to identify and map fundamental concepts, evidence, and gaps related to an area of interest. They assemble secondary data, critically evaluate research studies, and produce findings qualitatively or quantitatively. [22]

A systematic search is conducted followed by the collection and amalgamation of current knowledge, and the reporting the findings.

\section{Stage -1: Identifying the Research Question.}

The purpose of scoping reviews is to attain detailed and extensive results because they aim to detect all related literature regardless of study design. The central question of this literature review is posed as:

"How is immigrant patients' access to healthcare services affected by language barriers?"

\section{Stage -2: Identifying Relevant Studies}

This review study will identify, evaluate and combine relevant information from online databases; international, Commonwealth and state documents; journal articles and books. (Table 1) To discover all possible sources of information, the reference lists of all selected articles were scanned for relevant articles. 
Table 1. List of databases searched to identify literature for this synthesis.

\section{For published articles}

- MEDLINE

- PubMed

- Scopus

- CINAHL

- Web of Science

- Academic Search Complete

- Google

- Google Scholar

\section{TABLE 2: SEARCHED KEYWORDS IN DETAILS}

Table 2. Searched keywords in details.

- Language:

Language, Diversity, Linguistic, Non-English speaking, Communication

- Barrier:

Barrier, Impediment, Challenge, Obstacle, Hurdle, Difficult, Inequality, Obstruction

- Immigrant

Immigration, Migrant. Emigrant, Foreign-Born

- Access

Access, Healthcare Access, Healthcare Services Access, Healthcare Delivery, Healthcare Utilization, Accessibility

- Interpreter

Interpreter, Translator

A list of all possible key words (Table 2) for language, barrier, access, healthcare and immigrant was included. To reduce the number of studies to a more manageable number, considering the time required to examine each article identified, the search was limited to articles published from 2000 to 2019. Most studies identified were in the English language. The author decided to include only articles published in the English language in this review.

\section{Stage -3: Study Selection}

The preliminary search in the databases produced a significant number of both related and unrelated articles. An inclusion and exclusion criteria list considering the study question was created. (Table 3) Only full-text available articles focusing on language and communication barriers to healthcare access by immigrants (regardless of their country of origin), were selected. In the final refining step, only articles published in peer-reviewed journals were considered as peer-reviewed journals ensure a level of control and credibility.

\section{Stage -4: Data Charting}

An Excel spreadsheet consisting of a data charting form was developed with the following data: author(s), year of publication, country of study, study title, methodology used, population/sample size, major results and findings, and quality assessment rating for original research studies. A similar spreadsheet was also developed for literature review studies included in this review. 
Table 3. Searched Inclusion and Exclusion criteria used in the review.

\begin{tabular}{l|cc}
\hline Criteria & Inclusion & Exclusion \\
\hline Year published & Between 2000-2019 & Before 2000 \\
Language & English & Non-English \\
Online availability & Full text & Abstract only \\
Peer-reviewed & Yes & No \\
Focus & $\begin{array}{r}\text { Language and communication barriers between } \\
\text { health professionals and patients }\end{array}$ & Other barriers \\
Population & Immigrants & Non-migrants or unclear
\end{tabular}

\section{Stage -5: Reporting Results}

In scoping studies, the purpose is to develop a description of what research exists, or to create a thematic construction in order to present a broad view of research in a topic area. [21] In this systematic literature review, the author used the chart from stage 4 to further contextualize the collected information of the included articles based on language barriers and their effects on access to health care services, identified and coded within the article.

\section{ASSESSMENT OF QUALITY OF INCLUDED ARTICLES}

According to Levac et al. (2010), employing quality assessment tools could prevent false conclusions in regard to the nature and extent of the gaps identified in the study. [23] Therefore, after screening the full-text articles, all included articles were assessed using a quality assessment tool by Health Evidence ${ }^{\mathrm{TM}}$. [24] The quality of the articles was evaluated to safeguard the strength of the synthesis and their methodological quality. An overview of the quality assessment is shown in Table 4 and Table 5.

\section{RESULTS OF THE LITERATURE REVIEW}

A primary electronic search recognized 140 publications. From these articles, 109 records were selected based on title and abstract. These 109 records were further screened by reading the full texts and then reducing the number of articles within the scope to 20 original research articles. Four literature review articles were also identified that met the inclusion criteria making a final total of 24. No relevant grey literature was identified. All of the studies included in this synthesis were conducted on immigrants in developed countries and included a mix of health care providers' and patient's perspective. (Figure 1, Table 4 \& 5) 
Figure 1. Search flow for literature regarding language and communication barriers to accessing healthcare by immigrant patients.
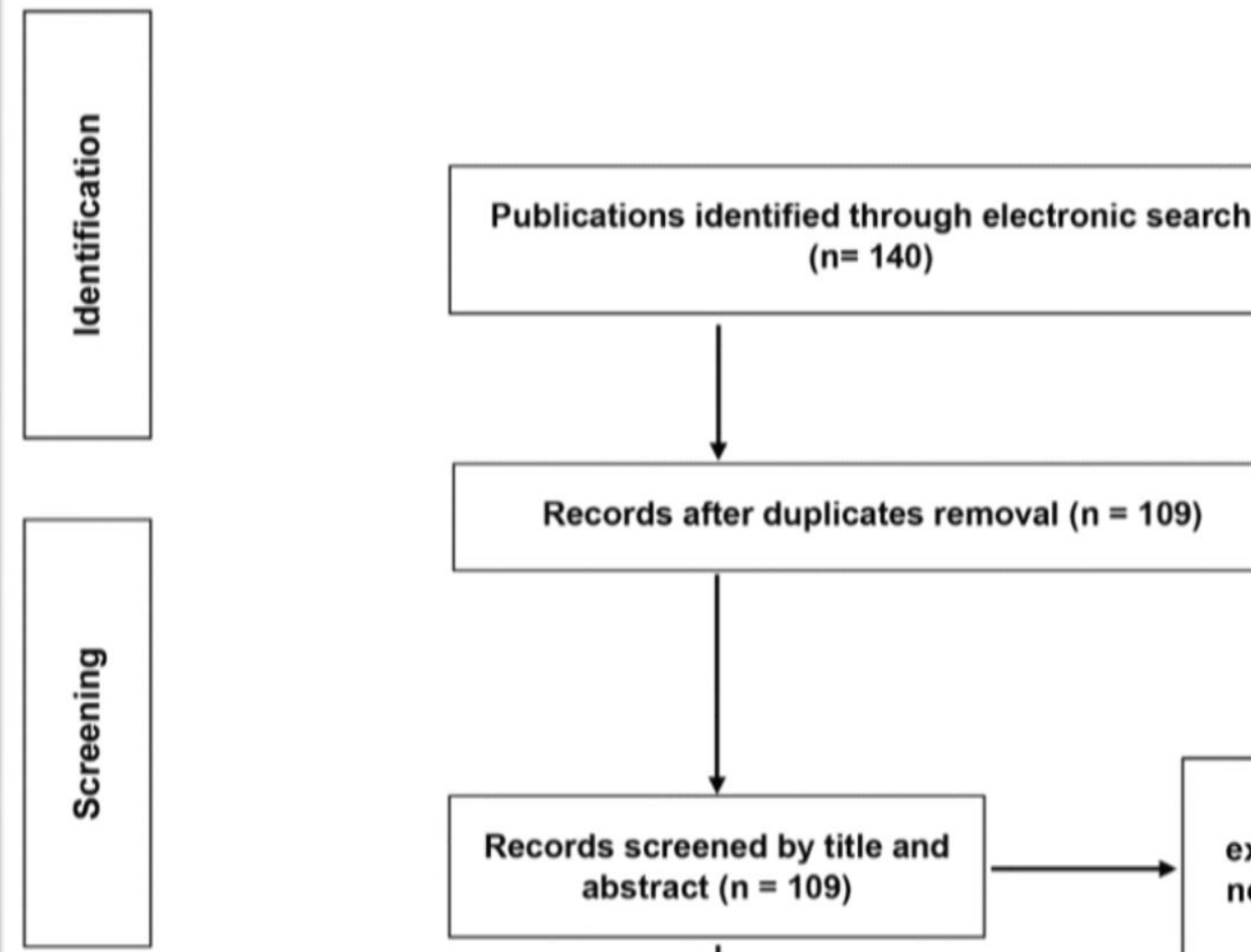

Records after duplicates removal $(n=109)$
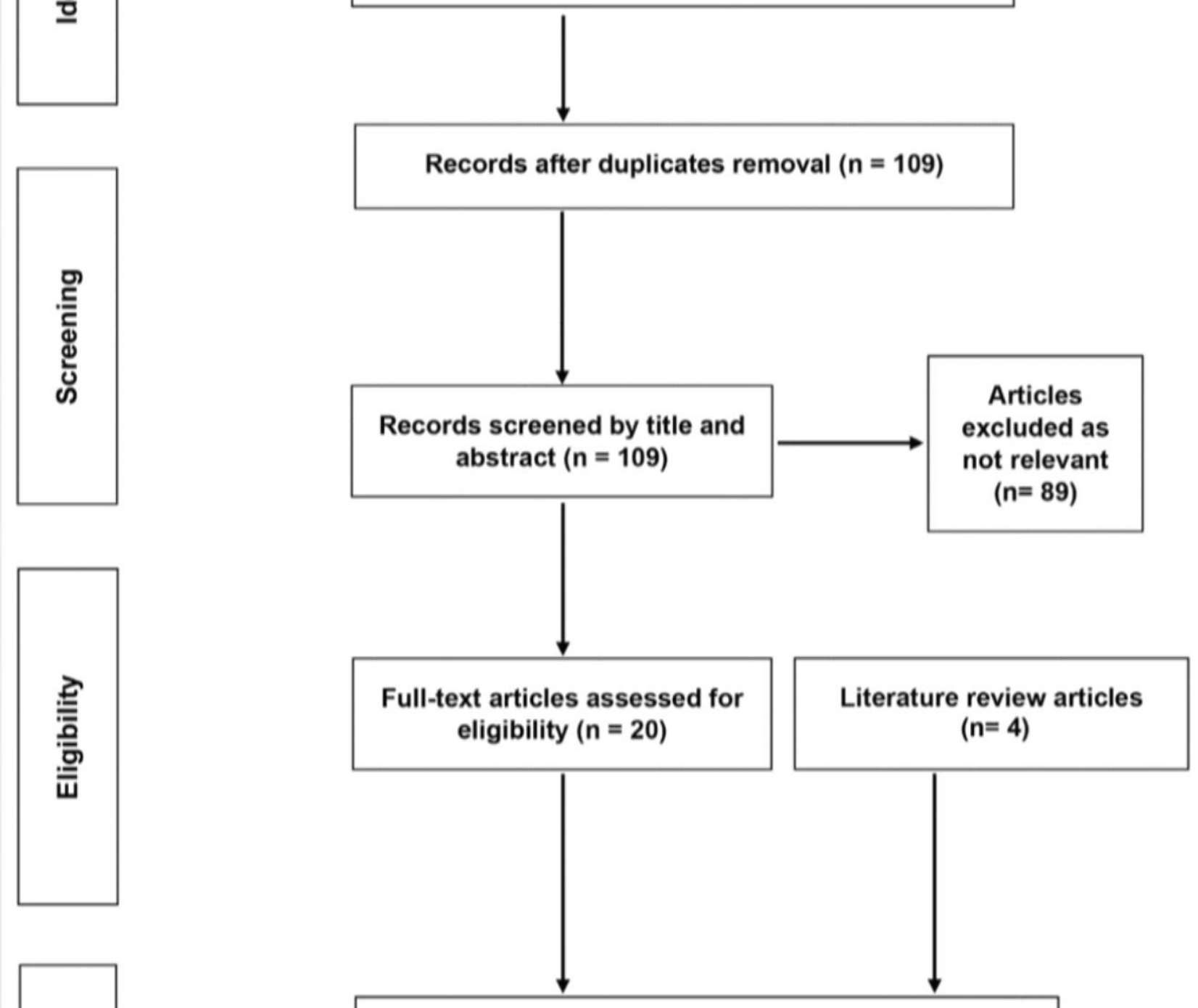

Full-text articles selected for the final analysis $(n=24)$

Original research: $(n=20)$

Literature review: $(n=4)$ 


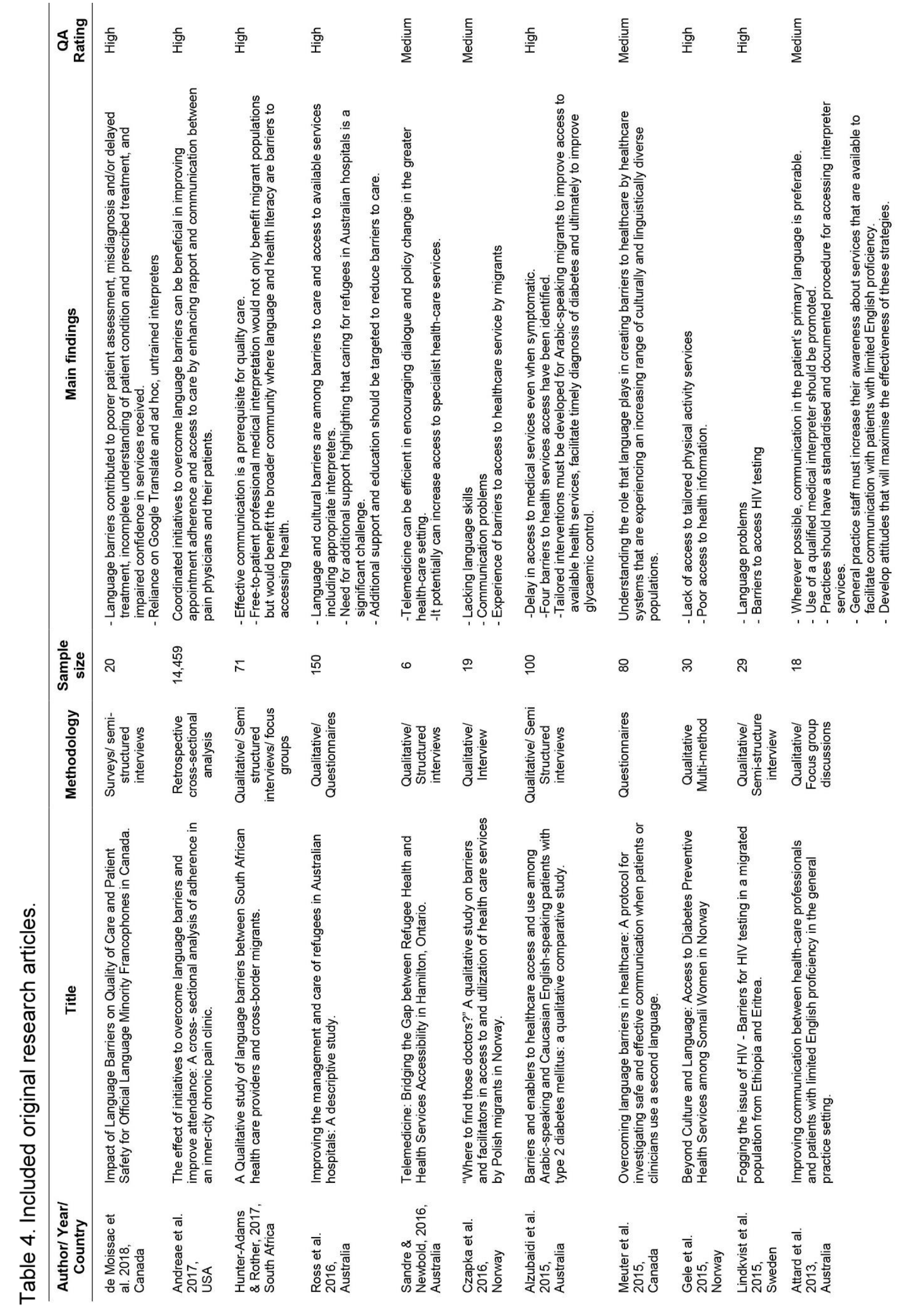




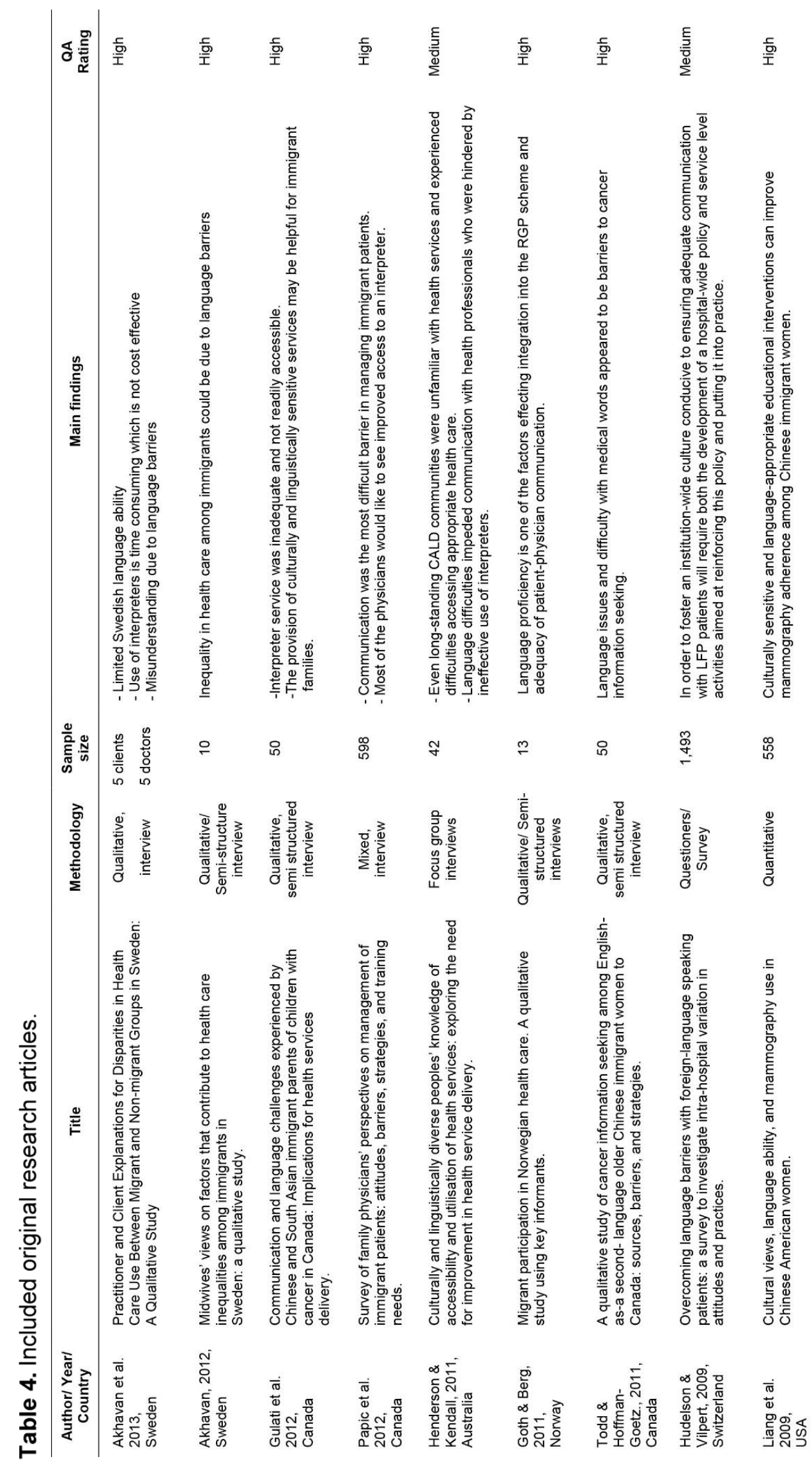




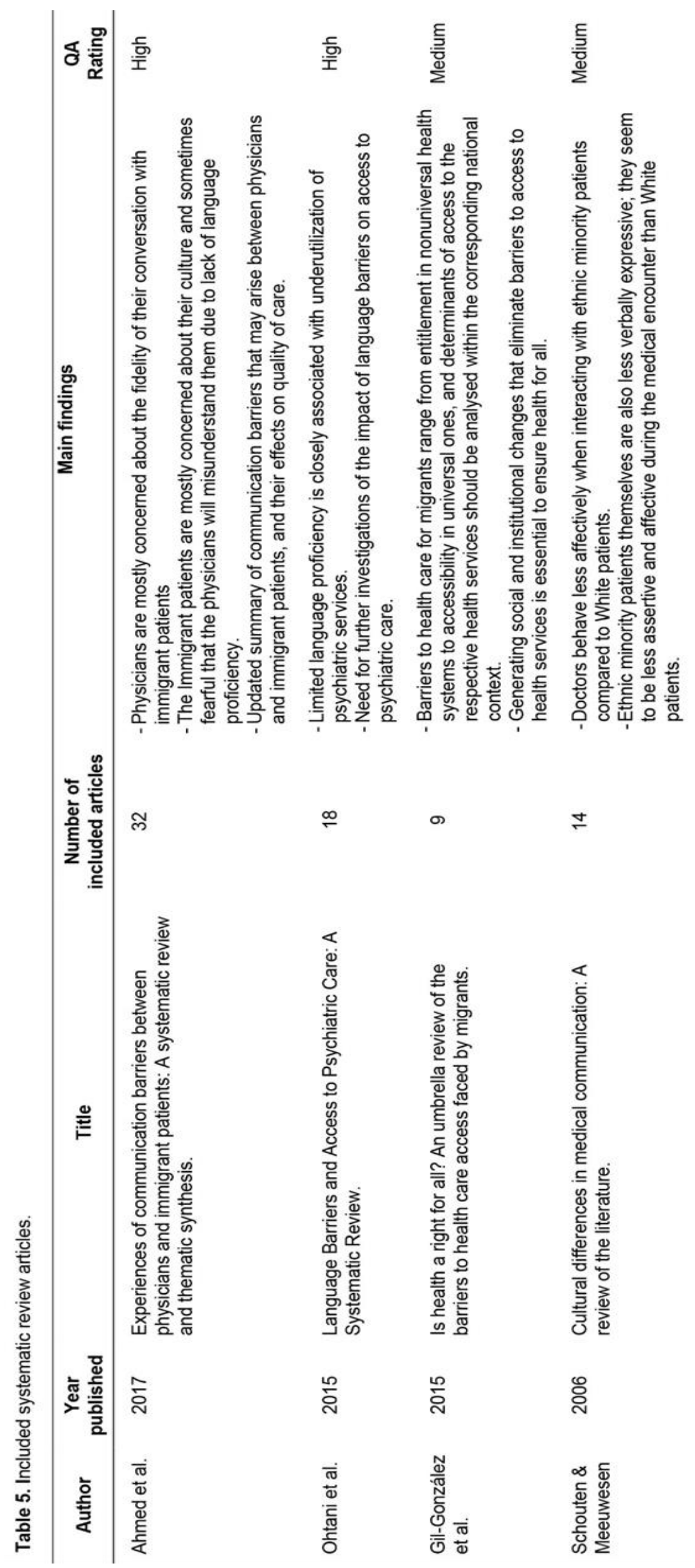


Table 6. Thematic data synthesis.

\begin{tabular}{|c|c|c|}
\hline Analytical themes & Descriptive themes & Reference number \\
\hline $\begin{array}{l}\text { Effects of language barriers on } \\
\text { access to healthcare services }\end{array}$ & $\begin{array}{l}\text { - Poorer patient assessment } \\
\text { - Misdiagnosis and/or delayed treatment } \\
\text { - Incomplete understanding of patient - } \\
\text { condition and prescribed treatment } \\
\text { - Impaired confidence in services received } \\
\text { - Quality of care } \\
\text { - Lack of access to healthcare information }\end{array}$ & $\begin{array}{c}6,9,12,13,15,16 \\
20,26 \\
28,29,30,34,36 \\
37,39,40\end{array}$ \\
\hline Role of interpreters & $\begin{array}{l}\text { - Reliance on Google Translate and ad hoc, } \\
\text { untrained interpreters } \\
\text { - Inadequate/ ineffective interpreter services } \\
\text { - Use of professional interpreters }\end{array}$ & $\begin{array}{c}6,20,26,34,36,39 \\
41\end{array}$ \\
\hline $\begin{array}{l}\text { Provision of immigrant-friendly } \\
\text { solutions }\end{array}$ & $\begin{array}{l}\text {-Appropriate training for health } \\
\text { professionals and migrants } \\
\text {-Ensure cultural competence in institutional } \\
\text { level } \\
\text { - Culturally and linguistically sensitive } \\
\text { approach \& policies }\end{array}$ & $26,29,40,41,42$ \\
\hline $\begin{array}{l}\text { Initiatives for overcoming language } \\
\text { barriers }\end{array}$ & Telemedicine & 31,43 \\
\hline
\end{tabular}

\section{THEMATIC SYNTHESIS OF THE RESULTS}

The synthesis of results identified four major themes within the included articles and references. (Table 6) The themes were identified by reading the articles and marking and underlining key phrases and concepts. The majority of the reviewed articles in this study cited language, communication, and access to health care services as problems for immigrant. Communication and language barriers were consistently reported in all included studies as an obstacle to accessing health care services.

\section{EFFECTS OF LANGUAGE BARRIERS ON ACCESS TO HEALTH CARE SERVICES}

Health care professionals in multicultural societies are faced with an increasing number of patients from diverse cultures and ethnicities. [25] Effective communication between health care professionals and patients is a prerequisite and important factor that influences quality of service provision and patient satisfaction. [6, 26-28] Communication could be verbal or non-verbal, such as body language, facial expression, gestures, clothing, eye contact, and tone of voice. Barriers to this communication can affect patients access to available information, understanding of the information received and their decisions to accept and adhere to some types of treatment and medications. [9, 12, 14, 28-30] It is also reported that language barriers can affect and delay access to healthcare services, even when symptomatic. [16] A review study conducted by Ohtani et al. (2015) with focus a on patients' access to psychiatric services has demonstrated a close association between migrant patients' limited language proficiency and underutilization of psychiatric services in the United States, Australia, Canada and the Netherlands. [7]

Identifying factors that can influence access to health care services may improve the delivery of these services to immigrant patients from different cultures. [5, 9] Studies have demonstrated that misinterpretation of the needs and wishes of immigrant patients or the information they received from health professionals can hinder their access to health care services. [12, 13, 15, 20] According to Akhavan (2012), miscommunication and misunderstanding may result from cultural diversity and from different cultural views, behaviour, and expectations. [12]

Goth and Berg (2011) believe that doctors are less affective when working with patients from ethnic minority groups where misunderstandings may occur. [13] Patients from 
ethnic minority groups in comparison to native citizens are less verbally communicative, less confident and less affective in their medical visits. [9, 10] These patients with inadequate language abilities require more time to explain their problems. [13] This can undoubtedly challenge the doctor-patient relationship and provision of effective quality services. [13, 14, 28, 31-33] Similarly, Ahmed et al. (2017) consider the significant impact of language and communication on the quality of interactions between immigrant patients and health professionals. The patients are occasionally afraid about possible misunderstanding because of language barriers. [33] Those patients facing language barriers are often unable to make an informed consent. They are at the increased risk of medical mistakes and adverse events, complications, and lack of confidentiality. [34]

Language barriers are even associated with underutilisation of health care services because these patients cannot adhere to their booked clinical arrangements and thus miss their required specialist care. [31] Patients often do not inform the medical practice about cancelling their appointments. This can result in ineffective arrangements, overbooking, and longer waiting time for bookings, underutilisation of clinic resources, and increasing practice costs. [35]

\section{ROLE OF INTERPRETERS}

Immigrant patients and healthcare staff use telephone interpreters which make it more challenging for patients to describe their symptoms and health status. It can often result in frequent use of emergency care. [13, 14] Patients occasionally use interpreters ineffectively [20], or seek help from Google Translate [34], next of kin, children, or friends to translate when they need assistance. [7, 14, 36] The involvement of unqualified translators, particularly the use of their children or a partner to interpret, may result in insecurities and uncertainty for care providers because they cannot trust unqualified interpreters' capabilities in appropriately transferring information. [7, 14, 15, 34, 37] Even the use of language translators from the same community as the immigrant patient may make an insecure situation for the patient in regard to the professional practice of the interpreter for keeping confidentiality. [13]

Karliner et al. (2007) demonstrate that employment of professional interpreters can increase the quality of health care service for these patients, facilitate good practice, [8,
38] and improve accessibility and health service use. [20, 26] In addition, Hunter-Adams and Rother (2017) pointed out that provision of free-to-patient professional medical interpretation can benefit both immigrant patients and the wider community when language barriers impede their access to health care services. [6] Similarly, Papic et al. (2012) has stressed the need to improve access to interpreters by health practitioners. [39]

\section{PROVISION OF IMMIGRANT-FRIENDLY SOLUTIONS}

In countries experiencing cultural and linguistic diversity, understanding effects of language barriers regarding access to health care services by health systems is essential. [9, 17] O'Donnell (2018) recommends that health care systems need to consider immigrant-friendly solutions such as provision of professional interpreters and appropriate trainings for both health professionals and immigrants. [39] Similarly, Hudelson and Vilpert, (2009) and Wolz (2015) agreed that health care providers should identify language barriers, provide adequate language services, and ensure cultural competence at the institutional level. [32, 40] Culturally and linguistically sensitive education and approaches can play a helpful role in improving the access to health care services by immigrant families. [16, 26, 29, 41 , 42]

\section{INITIATIVES FOR OVERCOMING LANGUAGE BARRIERS}

From all 24 reviewed articles, only Sandre and Newbold (2016) and Andreae et al. (2017) focused on use of coordinated initiatives to overcome language barriers and improving access to health care services. [31, 42] Sandre and Newbold (2016) proposed the use of communication technologies such as telemedicine to bridge the gap and effects of language barriers that hamper health care services accessibility for refugees. [43]

The term "telehealth" is identical to, and interchangeable with "telemedicine". [44] Telehealth is defined as the provision of healthcare services at a distance from a different geographical location, using information technology (ICT) and communication networks, mostly to respond to a shortage of health care facilities and professionals. [45] Telehealth may improve accessibility to primary, secondary and tertiary care for rural patients. [46] It can also be helpful in reducing emergency department visits, hospitalization, overall mortality [47] and in substantially saving health care costs. [48] 
In Australia, the Australian Medicare Benefits Schedule has covered video consultation by medical specialists to patients in rural and remote areas since June 2013. The federal government also supports the education of clinicians and promotes the uptake of telehealth $[49,50]$. Sandre and Newbold (2016) agree that telemedicine can effectively boost dialogue and policy change in the greater health-care setting. It potentially can also increase access to specialist health-care services. [42]

\section{DISCUSSION}

This systematic literature review of 24 articles that examined the impact of language barriers on access to health care services demonstrates consistent findings. These findings show clear association between insufficient language skills and affected access to health care services, regardless of where the study was conducted. Although the immigrants were entitled to access to health care services as equal to the native population, various factors such as communication and language have made access to healthcare services a challenge.

Because of mentioned barriers the process of consultation could be challenging and time-consuming, which may result in frustration for health professionals and for immigrant patients who are unable to communicate or understand the language of the host country. [33]

Some possible errors such as patient- healthcare staff miscommunication could arise and result in probable misdiagnosis. These patients would leave the health care centre confused and with low confidence in the care received and in the healthcare system in general. $[7,8]$ This negative experience and mistrust may continue and prevent them from seeking out future healthcare. [33]

Immigrant patients lacking communication skills with lowered access to healthcare services may also tend to utilise emergency services more often than native people. They are usually not required to make an appointment for emergency services. [7] Health care services are supposed to be equally accessible to legal immigrants as well as native citizens. Also, the provision of an effective health care service requires productive interaction between health care staff and patients. This level of communication is essential to healthcare professionals for understanding health status, uncovering symptoms, reaching right diagnoses, and planning proper treatments. [7]

This review supports the need for integrating cultural competency and awareness into health care systems. Immigrant-friendly strategies such as culturally and linguistically sensitive approaches and appropriate education for health staff and immigrants need to be considered by health systems. [38] Health care staff should be aware of immigrants' rights to access health care [38] and be updated about available services that ease communication with patients with language barriers. [26] Use of qualified medical interpreters as a practical solution for eliminating language barriers and bridging communication gaps is recommended by several studies, although it requires additional time and resources. [7, 14, $26,38]$

More research on language barriers in health care is still applicable. More studies need to be conducted to comprehensively identify the ways in which communication barriers affect health care, the efficiency of linguistic service interventions and the cost of languagecommunication barriers for the patients and health care system.

Finally, according to Sandre \& Newbold (2016), utilisation of communication technologies such as telemedicine could be an efficient tool for bridging the communication gap and increasing accessibility of healthcare services by immigrant patients. [43] The finding indicates the need for more studies and research in this area.

\section{FUTURE RESEARCH}

In light of the findings presented in this review, and the resultant discussion, the following recommendations are proposed for future research on immigrant patients' access to health care services:

- Application of telemedicine by employing bilingual general practitioners or nurses (Accessible via phone, or video call from all healthcare centres).

- Establishment of an online history-taking, triage, telemonitoring and referral system.

- Establishing mobile health services. 


\section{References}

1. International Organization for Migration. Global migration trends 2015 factsheet. 2016. Available:< http://gmdac.iom.int/global-migration-trendsfactsheet> (Accessed 20/04/2019)

2. Hjern A. Migration and public health in Sweden: The National Public Health Report. Scandinavian Journal of Public Health 2012; 40(9): 255-67.

3. United Nations. International migration report 2015: Department of Economic and Social Affairs.

Population Division 2016. Available:

<http://www.un.org/en/development/desa/populatio $\mathrm{n} /$ migration/publications/migrationreport/docs/Migrat ionReport2015_Highlights.pdf> (Accessed 15/04/2019)

4. Weitoft G, Ericsson, Ö, Loefroth E, Rosén M. Equal access to treatment? Population-based follow-up of drugs dispensed to patients with acute myocardial infarction in Sweden. European journal of clinical pharmacology 2008; 64: 417-24.

5. Gulliford M, Figueroa-Munoz J, Morgan M, Hughes

D, Gibson B, Beech R, Hudson M. What does 'access to health care' mean? Journal of Health Services Research \& Policy 2002; 7(3):186-8.

6. Hunter-Adams J, Rother HA. A Qualitative study of language barriers between South African health care providers and cross-border migrants. BMC Health Services Research 2017; 17:97.

7. Ohtani A, Suzuki T, Takeuchi H, Uchida H. Language Barriers and Access to Psychiatric Care: A Systematic Review. Psychiatric Services 2015; 66(8): 798-805.

8. Karliner LS, Jacobs EA, Chen AH, et al. Do professional interpreters improve clinical care for patients with limited English proficiency? A systematic review of the literature. Health Services Research 2007; 42: 727-754.

9. Meuter RFI, Gallois C, Segalowitz NS, Ryder AG, Hocking J. Overcoming language barriers in healthcare: A protocol for investigating safe and effective communication when patients or clinicians use a second language. BMC Health Services Research 2015; 15:371.

10. Schouten BC, Meeuwesen L. Cultural differences in medical communication: A review of the literature. Patient Education and Counselling 2006; 64: 21-34.
11. Green M. Language barriers and health of Syrian refugees in Germany. American Journal of Public Health 2017; 107(4), 486.

12. Akhavan S. Midwives' views on factors that contribute to health care inequalities among immigrants in Sweden: A qualitative study. International Journal of Equity Health 2012; $11: 47$.

13. Goth US, Berg JE. Migrant participation in Norwegian health care. A qualitative study using key informants. The European Journal of General Practice $2011 ; 17(1): 28-33$.

14. Håkonsen H, Lees K, Toverud EL. Cultural barriers encountered by Norwegian community pharmacists in providing service to non-Western immigrant patients. International Journal of Clinical Pharmacy 2014; 36(6): $1144-51$.

15. Lindkvist $P$, Johansson $E$, Hylander I. Fogging the issue of HIV - Barriers for HIV testing in a migrated population from Ethiopia and Eritrea. BMC Public Health 2015; 15(1): 82.

16. Alzubaidi H, MC Namara K, Browning C, Marriott J. Barriers and enablers to healthcare access and use among Arabic-speaking and Caucasian Englishspeaking patients with type 2 diabetes mellitus: a qualitative comparative study. BMJ open 2015; 5(11).

17. Gil-González D, Carrasco-Portiño M, Vives-Cases C, Agudelo-Suárez AA, Castejón Bolea R, Ronda-Pérez E. Is health a right for all? An umbrella review of the barriers to health care access faced by migrants. Ethnicity \& Health 2015; 20(5): 523-41.

18. Australian Bureau of Statistics. Migration, Australia, 2017-2018. Available <http://www.abs.gov.au/ausstats/abs@.nsf/mf/3412.0 $>$ (Accessed 5/04/2019)

19. Commonwealth of Australia. Australian multiculturalism for a new century: Towards Inclusiveness 2008. Available <http://www.multiculturalaustralia.edu.au/doc/dimia_ 3.doc> (Accessed 5/04/2019)

20. Henderson S, Kendall E. Culturally and linguistically diverse peoples' knowledge of accessibility and utilisation of health services: exploring the need for improvement in health service delivery. Australian Journal of Primary Health 2011; 17: 195-201. 
21. Arksey H, O'Malley L. Scoping studies: towards a methodological framework. International Journal of Social Research Methodology 2002; 8(1):19-32.

22. Armstrong R, Hall BJ, Doyle J, Waters E. 'Scoping the scope' of a Cochrane review, Journal of Public Health 2011; 33(1): 147-150.

23. Levac D, Colquhoun H, O'Brien KK. Scoping studies: advancing the methodology. Implementation Science 2010; 5: 69.

24. National Collaborating Centre for Methods and Tools. Health Evidence ${ }^{\mathrm{TM}}$ Quality Assessment Tool. Hamilton, ON: McMaster University. Available: <http://www.nccmt.ca/resources/search/275 > (Accessed 18/04/2019)

25. Statistics Netherlands. Migrants in The Netherlands. The Hague: Statistics Netherlands 2004. Available: $<$ https://www.cbs.nl//media/imported/documents/2005/17/2004-b-52pub.pdf?la=nl-nl> (Accessed 4/04/2019)

26. Attard M, MCArthur A, Riitano D, Aromataris E, Bollen C, Pearson A. Improving communication between health-care professionals and patients with limited English proficiency in the general practice setting. Australian Journal of Primary Health 2013; 21: 96-101.

27. Schyve PM. Language differences as a barrier to quality and safety in health care: The joint commission perspective. Journal of General Internal Medicine 2007; 22 (Suppl 2): 360-1.

28. Todd L, Hoffman-Goetz L. A qualitative study of cancer information seeking among English-as-asecond-language older Chinese immigrant women to Canada: Sources, barriers, and strategies. Journal of Cancer Education 201 1; 26(2): 333-340.

29. Ross L, Harding C, Seal A, Duncan G. Improving the management and care of refugees in Australian hospitals: A descriptive study. Australian Health Review 2016; 40: 679-685.

30. Czapka EA, Sagbakken M. "Where to find those doctors?" A qualitative study on barriers and facilitators in access to and utilization of health care services by Polish migrants in Norway. BMC Health Services Research 2016; 16(1): 460.

31. Andreae MH, White RS, Yan Chen K, Nair S, Hall C, Shaparin N. The effect of initiatives to overcome language barriers and improve attendance: A cross- sectional analysis of adherence in an inner-city chronic pain clinic. Pain Medicine 2017; 18: 265-274.

32. Wolz MM. Language barriers: Challenges to quality healthcare. International Journal of Dermatology 2015; 54: 248-250.

33. Ahmed S, Lee S, Shommu N, Rumana N, Turin T. Experiences of communication barriers between physicians and immigrant patients: A systematic review and thematic synthesis. Patient Experience Journal 2017; 4(1): 122-140.

34. de Moissac D, Bowen S. Impact of language barriers on quality of care and patient safety for official language minority Francophones in Canada. Journal of Patient Experience 2018; 6(1): 24-32.

35. Lynch ME, Campbell F, Clark AJ, et al. A systematic review of the effect of waiting for treatment for chronic pain. Pain 2018; 136(1-2): 97-116.

36. Akhavan S, Karlsen S. Practitioner and client explanations for disparities in health care use between migrant and non-migrant groups in Sweden: A qualitative study. Journal of Immigrant and Minority Health 2013; 15(1): 188-97.

37. Gele AA, Torheim LE, Pettersen KS, Kumar B. Beyond culture and language: Access to diabetes preventive health services among Somali women in Norway. Journal of Diabetes Research 2015; 2015.

38. O'Donnell CA. Health Care Access for Migrants in Europe. Oxford Research Encyclopaedia of Global Public Health 2018. Available <http://oxfordre.com/publichealth/view/10.1093/acre fore/9780190632366.001.0001/acrefore9780190632366-e-6> (Accessed 11/04/2019)

39. Papic O, Malak Z, Rosenberg E. Survey of family physicians' perspectives on management of immigrant patients: attitudes, barriers, strategies, and training needs. Patient Education and Counseling 2012; 86(2): 205-209

40. Hudelson P, Vilpert S. Overcoming language barriers with foreign-language speaking patients: a survey to investigate intra-hospital variation in attitudes and practices. BMC Health Services Research 2009; 15(9):187.

41. Gulati S, Watt L, Shaw N, Sung L, Poureslami IM, Klaassen R, Dix D, Klassen AF. Communication and language challenges experienced by Chinese and south Asian immigrant parents of children with cancer 
in Canada: Implications for health services delivery. Pediatric Blood \& Cancer 2012; 58(4): 572-578.

42. Liang W, Wang J, Chen MY, Feng S, Yi B,

Mandelblatt JS. Cultural views, language ability, and mammography use in Chinese American women. Health Education \& Behavior 2009; 36(6): 1012-1025.

43. Sandre AR, Newbold K B. Telemedicine: Bridging the gap between refugee health and health services accessibility in Hamilton, Ontario. Canada's Journal on Refugees 2016; 32: 3.

44. Fatehi F, Wootton R. Telemedicine, telehealth or ehealth? A bibliometric analysis of the trends in the use of these terms. Journal of Telemedicine and Telecare 2012; 18: 460-464

45. Armstrong BK, Gillespie JA, Leeder SR, Rubin GL, Russell LM. Challenges in health and health care for Australia. Medical Journal of Australia 2007; 187: 485-9.

46. Bashshur RL, Shannon GW. National telemedicine initiatives: essential to healthcare reform. Telemedicine Journal and E Health 2009; 15(6): 600-10.

47. Steventon A, Bardsley M, Billings J, Dixon J, Doll H, Hirani $S$, et al. Effect of telehealth on use of secondary care and mortality: findings from the Whole System Demonstrator cluster randomised trial. British Medical Journal 2012; 344: e3874

48. Cusack CM, Pan E, Hook JM, Vincent A, Kaelber DC, Middleton $B$. The value proposition in the widespread use of telehealth. Journal of Telemedicine and Telecare 2008; 14(4): 167-8.

49. Australian College of Rural and Remote Medicine. Technology directory. Available $<$ http://www.ehealth.acrrm.org.au/technologydirectory> (Accessed 17/04.2019)

50. Royal Australian College of General Practitioners. Hardware and software. Available < https://www.racgp.org.au/running-apractice/technology/business-technology/hardwareand-software-requirements> (Accessed 17/04/2019) 